\section{Análises espaciais na identificação das áreas de risco para a esquistossomose mansônica no Município de Lauro de Freitas, Bahia, Brasil}

\author{
Identification of schistosomiasis risk areas using \\ spatial analysis in Lauro de Freitas, Bahia \\ State, Brazil
}

\author{
1 Escola de Medicina \\ Veterinária, Universidade \\ Federal da Bahia, Salvador, \\ Brasil. \\ 2 Departamento de Vigilância \\ à Saúde, Secretaria \\ Municipal de Saúde de Lauro \\ de Freitas, Lauro de Freitas, \\ Brasil. \\ 3 Departamento de Ciências \\ Exatas, Universidade \\ Estadual Paulista, \\ Jaboticabal, Brasil. \\ 4 Centro de Pesquisa Gonçalo \\ Moniz, Fundação Oswaldo \\ Cruz, Salvador, Brasil. \\ Correspondência \\ L. L. Cardim \\ Departamento de Medicina \\ Veterinária Preventiva, \\ Escola de Medicina \\ Veterinária, Universidade \\ Federal da Bahia. \\ Av. Adhemar de Barros 500, \\ Salvador, BA \\ 40170-970, Brasil. \\ lucianacardim@yahoo.com.br
}

\section{Abstract}

The spread of schistosomiasis mansoni defies efforts by Brazil's Unified National Health System, thus demonstrating the need to reassess endemic control programs in the country. The aim of this study was to demarcate geographic areas at risk of schistosomiasis in Lauro de Freitas, Bahia State, Brazil, and to establish the epidemiological and socioeconomic profile of the disease in this municipality (county). Kernel density estimator exploratory analysis was used for visual identification of areas at risk. Kulldorff \& Nagarwalla's spatial analysis was used to obtain statistically significant clusters and to measure risk. These technologies identified four risk areas for schistosomiasis. Clusters identified within the risk areas were characterized by lower socioeconomic conditions. Multiple correspondence analyses showed a distinct profile for positive patients in the primary cluster. The techniques employed here represent an important methodological acquisition for tracking and controlling schistosomiasis in Lauro de Freitas.

Schistosomiasis Mansoni; Spatial Analysis; Risk Zone
Luciana Lobato Cardim 1,2 Antonio Sergio Ferraudo 3 Selma Turrioni Azevedo Pacheco 2 Renato Barbosa Reis 4 Marta Mariana Nascimento Silva 1 Deborah Daniela M. Trabuco Carneiro 1 Maria Emilia Bavia 1

\section{Introdução}

A esquistossomose mansônica é uma das principais doenças parasitárias de veiculação hídrica no mundo, estimando-se a existência de aproximadamente 200 milhões de pessoas infectadas na América do Sul, Ásia e África 1,2. Causada pelo trematódeo digenético Schistosoma mansoni, tem o homem como principal reservatório e caramujos do gênero Biomphalaria como hospedeiros intermediários ${ }^{3}$.

No Brasil, essa doença ainda se constitui em um dos mais sérios problemas de saúde pública, tendo em vista seu potencial de expansão. Atualmente, a presença de portadores da parasitose é observada em 19 das 27 Unidades da Federação, distribuídas em todas as regiões do país, com áreas de concentração no Nordeste e no Estado de Minas Gerais 4.

Na Bahia, a doença é notificada em 65\% (271/417) dos municípios, com média de 165,8 internações/ano, 40,2 óbitos/ano e prevalência de 5,4\% 5. O Município de Lauro de Freitas vem se transformando em uma preocupação dos responsáveis pelo Programa de Controle da Esquistossomose, por ser classificado pela Secretaria de Saúde do Estado da Bahia como de transmissão focal para a doença e por apresentar-se em plena ascensão econômica, atraindo intensos movimentos migratórios, com ocupação populacional descontrolada no espaço urbano e com con- 
sequentes distúrbios ambientais que aumentam o risco da expansão geográfica da endemia.

A vigilância epidemiológica da esquistossomose mansônica tem como objetivos principais: reduzir a prevalência da infecção em áreas endêmicas; reduzir a morbidade e a mortalidade; evitar a ocorrência de formas graves da patologia e reduzir o risco de expansão 6,7. No entanto, a diversidade dos fatores que envolvem a transmissão da doença dificulta de forma marcante o seu controle pelos serviços de saúde, deixando clara a necessidade do desenvolvimento de novas ferramentas capazes de congregar aspectos sociais, ambientais e identificadores de riscos que permitam a detecção de surtos epidêmicos e o seu acompanhamento no tempo e no espaço, minimizando os possíveis danos à população exposta ${ }^{8}$.

Nesse contexto, obedecendo à metodologia do enfoque de risco preconizado pela Organização Mundial da Saúde (OMS), que objetiva a detecção de grupos específicos para o direcionamento das ações de saúde com maior eficiência na aplicação de recursos públicos em países subdesenvolvidos e em desenvolvimento econômico, surgem as geotecnologias e as análises espaciais, capazes de delimitar áreas homogêneas e mensurar o risco de infecção dentro dessas áreas 9,10.

Assim, com base na identificação do perfil da distribuição espaço-temporal da esquistossomose mansônica no Município de Lauro de Freitas, este trabalho tem como objetivo delimitar, por meio das análises espaciais, suas áreas geográficas de risco.

\section{Material e métodos}

O estudo foi conduzido no Município de Lauro de Freitas, situado na região metropolitana de Salvador, a aproximadamente 100m de altitude acima do nível do mar e com uma população de 156.936 habitantes, distribuída em 7 distritos sanitários e 111 setores censitários 11 . O município apresenta uma dinâmica econômica importante para a economia estadual, baseada no comércio e no turismo.

O período de estudo compreende os anos de 2006 a 2008, tendo como definição de caso para a esquistossomose mansônica os indivíduos positivos para a doença, diagnosticados por meio da campanha de controle desta endemia no município usando-se o exame coproparasitológico pelo método Kato-Katz. Os casos esquistossomóticos foram descritos segundo as variáveis: endereço de residência, localidade, idade, sexo, tratamento, mês e ano da detecção do caso.
O levantamento dos dados foi realizado por meio de consulta direta a cada uma das fichas de notificação existentes no Departamento de Vigilância à Saúde da Secretaria Municipal de Saúde de Lauro de Freitas (VISAU/LF). As fichas com dados incompletos $(20,8 \%)$ foram eliminadas.

Para a localização espacial dos endereços foi utilizado o receptor GPS (Global Position System), cuja captura de coordenadas foi feita em sistema de projeção UTM (Universal Transversa de Mercador) e de referência elipsoidal SAD69, etapa que contou com o auxílio dos agentes de endemias do município.

Os dados socioeconômicos e sociodemográficos por setor censitário foram coletados no Instituto Brasileiro de Geografia e Estatística (Censo Demográfico 2000. http://www.ibge.gov.br). As coleções hídricas foram obtidas usando-se a digitalização da base cartográfica de Lauro de Freitas em escala 1:2.000, realizada pela Companhia de Desenvolvimento Urbano do Estado da Bahia (CONDER). As informações mensais de precipitação pluviométrica foram fornecidas pelo Instituto Nacional de Meteorologia (INMET).

O cálculo da distância média entre os casos positivos e as coleções hídricas presentes no município foi realizado utilizando-se o programa ArcGIS, versão 9.1 (http://www.esri.com/softwa re/arcgis/index.html). A análise de correlação de Pearson, com nível de 5\% de significância, foi realizada com a finalidade de verificar a existência de correlação linear entre os casos de esquistossomose mansônica e a precipitação pluviométrica.

\section{Análises espaciais}

Os setores censitários foram considerados unidades básicas de análise por constituírem unidades espaciais com o maior nível de desagregação disponível e, portanto, relativamente mais homogêneas, para os quais há disponibilidade de dados censitários 12 .

As análises de densidade para a identificação de "áreas quentes" foram feitas por meio do estimador de densidade de Kernel, implementado na extensão Spatial Analyst do programa ArcGIS, versão 9.2, utilizando a função quártica de suavização ${ }^{13}$. Foi definida uma largura de banda de $1 \mathrm{~km}$, a partir da qual foram construídas superfícies para os casos, para a população e para a razão entre as densidades de casos e população (razão de Kernel), sendo esta última uma aproximação das áreas de risco, uma vez que seus valores encontram-se ponderados pela relação caso/ população.

Para a identificação de aglomerados espaçotemporais estatisticamente significantes foi utilizado o método de varredura proposto por Kull- 
dorff \& Nagarwalla 14 , processado pelo programa SaTScan, versão 8.0 (http://www.satscan.org, Estados Unidos). Para a distribuição dos casos foi considerada a de Poisson. Foi apontada como $30 \%$ a porcentagem máxima da população total exposta ao risco.

\section{Análise multivariada}

A análise de correspondência múltipla foi utilizada, em complemento às análises espaciais, para investigar a existência de associação entre as variáveis categóricas: grupo etário, sexo, ano da detecção do caso, precipitação pluviométrica, distância média entre os casos de esquistossomose e as coleções hídricas e aglomerados. Os dados foram processados pelo programa Statistica, versão 7.0 (Statsoft Inc.; http:/ /www.statsoft. com) e os resultados foram representados graficamente no mapa perceptual/intuitivo, cuja interpretação é baseada na localização dos pontos e nas medidas de similaridades criadas conforme padronização da tabela de contingência pelo valor do qui-quadrado de cada célula 15 .

\section{Aspectos éticos}

A utilização dos dados secundários foi autorizada pela diretoria do VISAU/LF, por meio do Termo de Consentimento de Utilização de Dados, em acordo com as normas éticas da Comissão Nacional de Ética em Pesquisa (CONEP).

\section{Resultados}

Entre os anos de 2006 e 2008 foram notificados 1.006 casos de esquistossomose mansônica no Município de Lauro de Freitas, sendo 37,6\% (378/1.006) no ano de 2006, 25,8\% (260/1.006) no de 2007 e 36,6\% (368/1.006) no ano de 2008. A média de idade foi de 30,9 anos, variando de 3 a 80 anos. O sexo masculino representou $63,4 \%$ (638/1.006) dos casos. Do total de indivíduos positivos, 66,8\% (672/1.006) foram tratados com Praziquantel. De acordo com a análise de correlação de Pearson não foi observada associação entre as variáveis casos de esquistossomose e precipitação pluviométrica ( $\mathrm{R}=0,1338 ; \mathrm{p}=0,2183$ ).

Como não foi possível localizar o endereço de 209 registros, nas análises espaciais foram utilizados apenas 797 casos (Figura 1a). As regiões com tons mais escuros, obtidas por meio do estimador de Kernel, mostram as áreas com maior densidade de casos (Figura 1b) e de população (Figura 1c). Os resultados da análise da razão de Kernel apontam a existência de quatro áreas principais de risco para a esquistossomose man- sônica no município: a primeira, caracterizada visualmente pelo aglomerado com maior densidade de casos, encontra-se no distrito sanitário de Portão; a segunda e a terceira encontram-se no distrito de Caji-Picuaia e a quarta no distrito de Ipitanga (Figura 1d).

A análise de varredura espaço-temporal confirmou os aglomerados espaciais identificados por Kernel (Figura 2). Os resultados dessa análise apontam a existência de quatro grupos de aglomerados com forte significância estatística ( $p=0,001$ ), sendo o primeiro considerado o mais verossímil (ou primário) e os demais secundários (Figura 2b).

O aglomerado de maior razão de probabilidade de ocorrência, designado cluster primário, foi detectado no ano de 2006, com uma área de aproximadamente $1,5 \mathrm{~km}^{2}$, distribuída em cinco setores censitários do distrito sanitário de Portão. Esse aglomerado englobou 11,3\% da população total do município e abrigou 35,8\% dos casos de esquistossomose georreferenciados. Para o período, sob a hipótese nula da casualidade, eram esperados cerca de 16,0 casos da enfermidade, e foram notificados 285, numa relação entre observados e esperados de 17,8. A taxa de risco anual foi estimada em 3.148,7 para cada grupo de 100 mil habitantes e o risco relativo em 27,2 .

Os aglomerados secundários foram detectados no ano de 2008. O aglomerado designado cluster secundário I englobou dez setores censitários do distrito sanitário de Ipitanga, com uma área de aproximadamente $1,8 \mathrm{~km}^{2}$ e composto por 17.357 residentes $(11,3 \%$ da população total do município). O número de casos encontrados nessa área foi de 151, esperando-se sob a hipótese nula o número de 31,3 casos, numa relação entre observados e esperados de 4,8. A região apresentou risco relativo estimado em 5,7.

O aglomerado designado cluster secundário II abrangeu uma área de aproximadamente $0,2 \mathrm{~km}^{2}$, referente a dois setores censitários do distrito sanitário de Caji-Picuaia e envolveu 2.662 pessoas ( $1,7 \%$ da população total), apresentando 45 casos contra os 4,8 esperados sob hipótese nula, numa relação entre observados e esperados de 9,4. O risco relativo foi estimado em 9,9.

Por sua vez, a região do aglomerado designado cluster secundário III envolveu apenas um setor censitário do distrito sanitário de Caji-Picuaia, com aproximadamente $2,4 \mathrm{~km}^{2}$ de área, 1.355 habitantes $(0,9 \%$ da população total) e risco relativo de 5,8. Nessa área foram notificados 14 casos contra os 2,4 esperados sob hipótese nula, numa relação entre observados e esperados de 5,7.

Os setores censitários pertencentes às áreas com aglomerados apresentam indicadores socioeconômicos mais baixos quanto à forma de 
Figura 1

Distribuição espacial dos casos de esquistossomose mansônica diagnosticados e análise de densidade de Kernel. Município de Lauro de Freitas, Bahia, Brasil, 2006 a 2008.

1a) Distribuição pontual dos casos

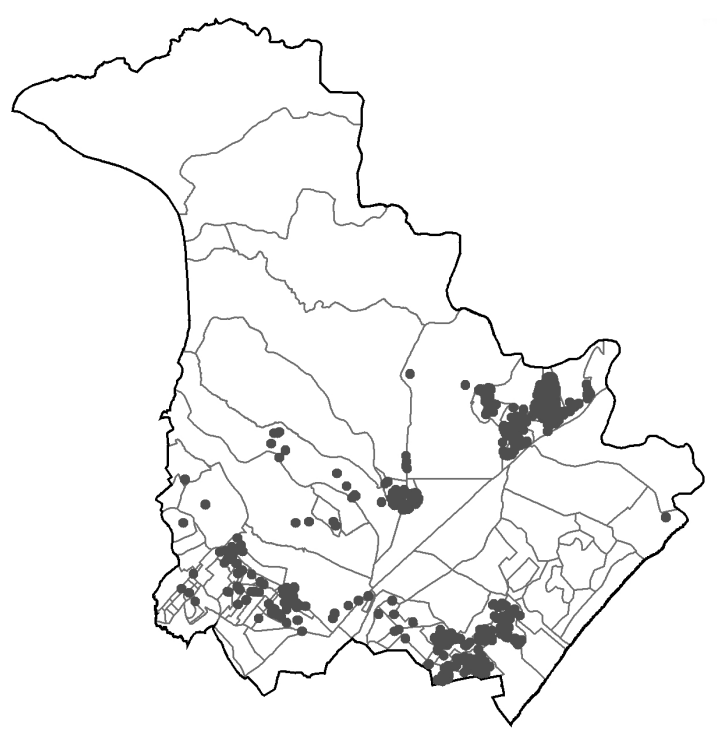

1c) Kernel: densidade da população

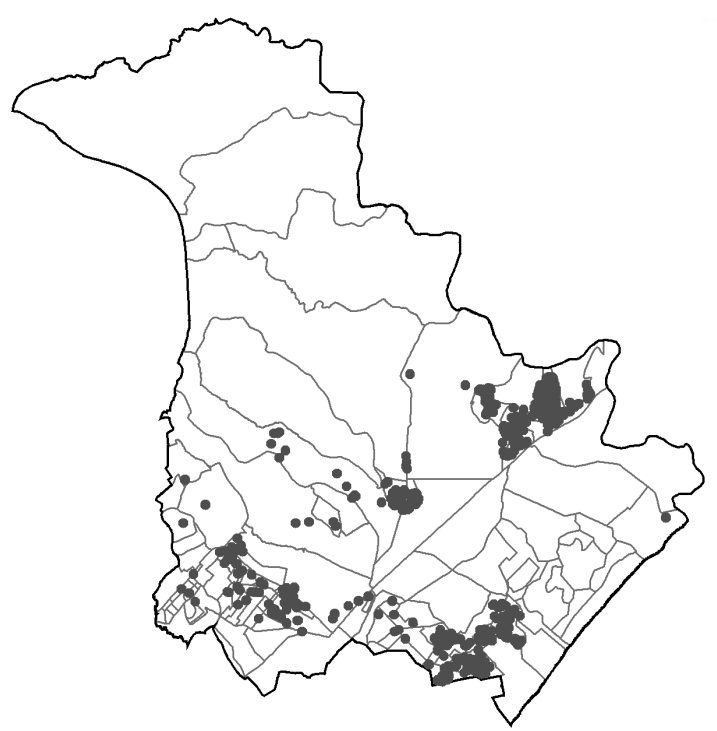

Alta densidade

Baixa densidade 1b) Kernel: densidade de casos

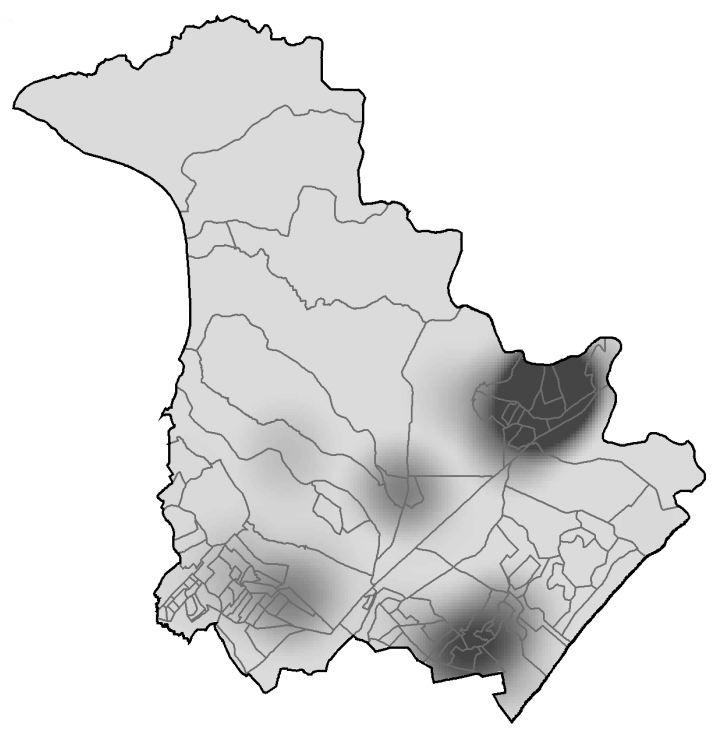

1d) Razão de Kernel
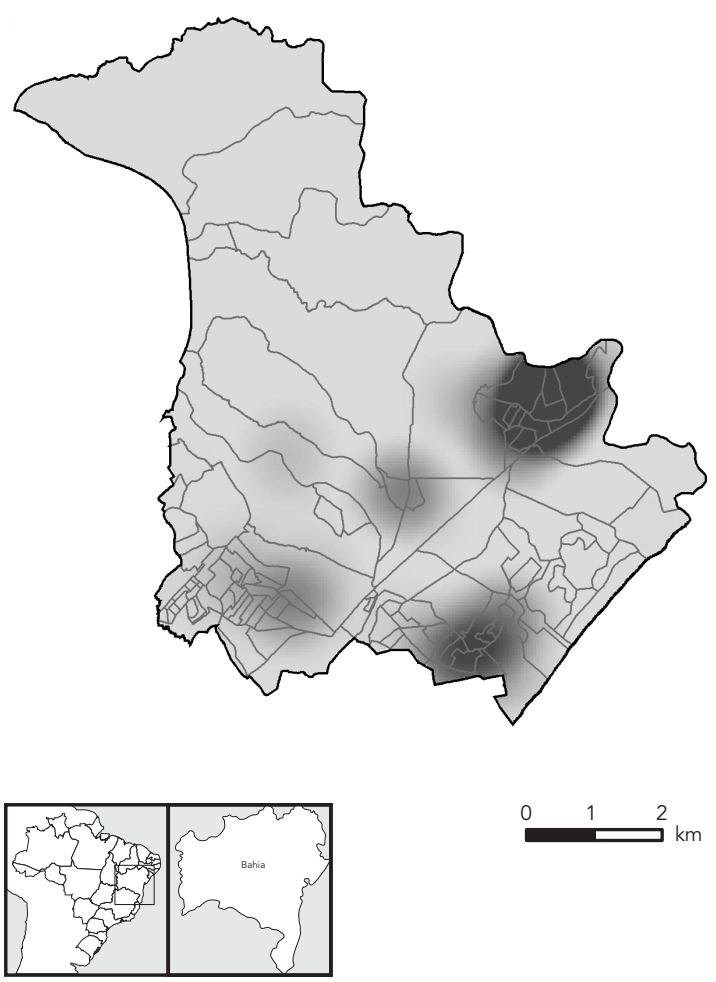
2a) Razão de Kernel
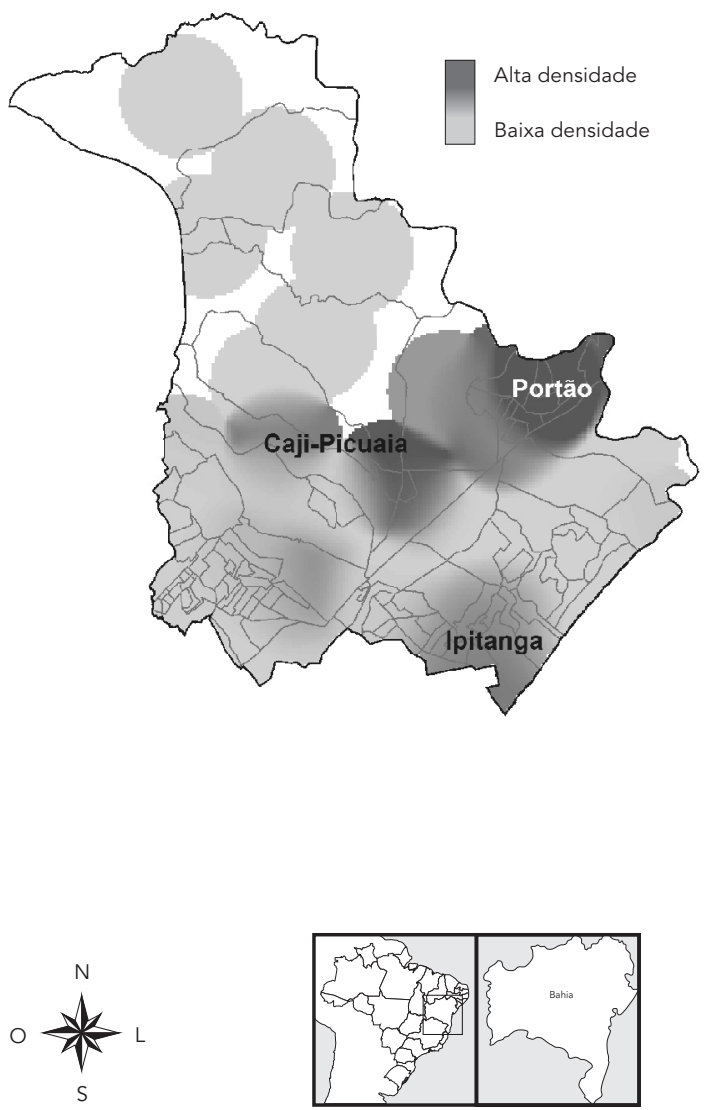

2b) Análise de varredura espaço-temporal

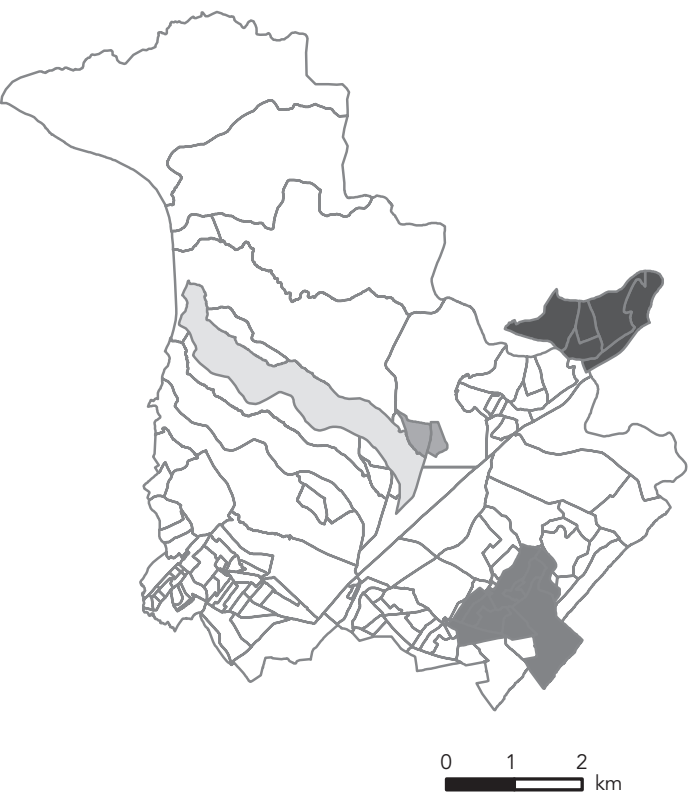

Cluster primário

Cluster secundário I

Cluster secundário II

Cluster secundário III abastecimento de água, à presença de sanitário, ao destino do lixo, à proporção de pessoas alfabetizadas, ao grau de escolaridade e ao rendimento mensal das pessoas responsáveis pelos domicílios (Tabela 1).

O mapa perceptual, resultante da análise de correspondência múltipla, permitiu uma distinção de quatro grupos de variáveis com características próprias (Figura 3), sendo o mais importante o grupo discriminado à esquerda na dimensão 1, pois possui correspondências específicas, envolvendo as variáveis idade de 0 a 14 anos, precipitação pluviométrica maior que 200mm, ano de 2006 e cluster primário. O segundo grupo contém variáveis com correspondências entre idade de 15 a 29 anos, distância para água de 51 a 100m, sexo feminino, sexo masculino, precipitação pluviométrica de 0 a $100 \mathrm{~mm}$, idade maior que 29 anos, distância para a água maior que $100 \mathrm{~m}$, precipitação pluviométrica de 101 a 200mm e distância para a água de 0 a 50m, que por localizarem-se na região central do mapa perceptual não apresentam características específicas. O terceiro grupo tem variáveis com correspondências entre cluster secundário e ano de 2008, e finalmente o quarto grupo contém correspondências entre as variáveis não cluster e ano de 2007. A qualidade do mapa perceptual é expressa pela inércia de cada dimensão, que juntas condensaram 35,99\% (20,82\% na dimensão 1 e $15,17 \%$ na dimensão 2).

As associações observadas entre os casos positivos que compuseram o aglomerado primário e ano de 2006, aglomerado secundário e ano de 2008 e sem aglomerado e ano de 2007 já eram esperadas, pois a análise de correspondência 
Tabela 1

Características socioeconômicas das áreas de clusters identificadas por meio da análise de varredura espaço-temporal dos casos de esquistossomose mansônica. Município de Lauro de Freitas, Bahia, Brasil, 2006 a 2008.

\begin{tabular}{lcc}
\hline Variáveis & Cluster & Não cluster \\
\hline Proporção de domicílios & & 86,4 \\
Abastecimento de água via rede geral & 80,6 & 8,2 \\
Abastecimento de água via poço ou nascente & 9,3 & 93,3 \\
Com sanitário & 90,8 & 3,8 \\
Sem sanitário & 8,3 & 87,7 \\
Destino do lixo coletado & 83,1 & 5,0 \\
Destino do lixo jogado em terreno baldio & 12,4 & 86,9 \\
Proporção de pessoas responsáveis pelos domicílios & 81,1 & 10,2 \\
Alfabetizadas & 18,0 & 23,2 \\
Não alfabetizadas & 15,4 & 30,6 \\
Curso mais elevado (Ensino Médio) & 42,2 & 51,6 \\
Curso mais elevado (Ensino Fundamental) & 60,1 & 32,3 \\
Rendimento mensal até 3 salários mínimos & 20,1 & \\
Rendimento mensal maior que 3 salários mínimos & & \\
\hline
\end{tabular}

Figura 3

Mapa perceptual mostrando padrões de correspondências entre as variáveis utilizadas na avaliação da esquistossomose mansônica. Município de Lauro de Freitas, Bahia, Brasil, 2006 a 2008.

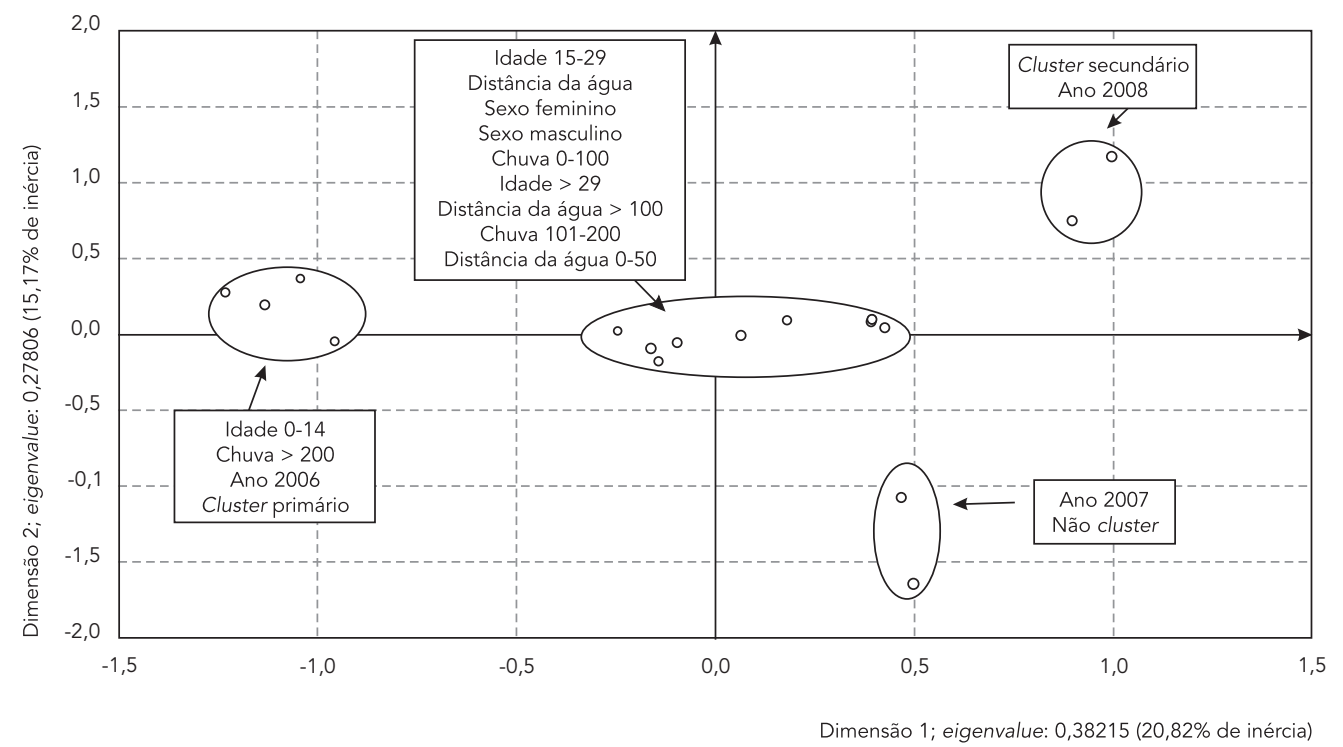

múltipla foi feita de acordo com os resultados da análise espaço-temporal que já havia identificado essa correlação.
Os maiores valores do qui-quadrado nas diferenças positivas entre cada valor observado e esperado no grupo aglomerado primário foram 
para as variáveis ano de $2006(259,9)$, idade de 0 a 14 anos $(31,3)$ e precipitação pluviométrica maior que 200mm $(29,6)$ (Tabela 2), o que confirma a associação observada à esquerda da dimensão 1 do mapa perceptivo (Figura 3). O sinal (+) indica valores positivos da diferença entre observado e esperado.

\section{Discussão}

No período estudado foram diagnosticados 1.006 indivíduos positivos para a doença, no entanto, acredita-se que o número de casos seja maior do que o observado, pois a metodologia recomendada pela OMS 16 e utilizada no Programa de Controle da Esquistossomose do município baseada apenas em uma lâmina por amostra de fezes pelo método Kato-Katz apresenta baixa sensibilidade diagnóstica em áreas onde a doença é de pouca gravidade, em que os pacientes positivos eliminam pequenas quantidades de ovos 17,18, como ocorre no Município de Lauro de Freitas. Essa observação é muito importante porque, ainda que as infecções leves, não diagnosticadas, não sejam responsáveis pelo aparecimento da patologia grave, podem vir a ser responsáveis pela persistência da transmissão da doença no município.

Em relação à faixa etária, observou-se que $75 \%$ dos casos ocorreram na faixa economicamente ativa. No entanto, esses casos, possivelmente, não prejudicaram a economia local, uma vez que foram todos assintomáticos. Neste estudo, a infecção pelo $S$. mansoni entre os homens foi aproximadamente duas vezes superior que a encontrada entre as mulheres, corroborando com os achados de outros autores, como Nomura et al. ${ }^{19}$ que identificaram 28 pacientes do sexo masculino entre os 31 examinados no Município de Parauapebas, Pará; e Vasconcelos et al. ${ }^{20}$ que verificaram 21 homens do total de 38 indivíduos examinados no Município de Sabará, Minas Gerais. O tipo de atividade econômica a que se encontra inserida a população de Lauro de Freitas afasta a possibilidade da infecção entre os homens ser maior em virtude das atividades laborais. A maior prevalência desse grupo pode ser decorrente dos hábitos culturais e de lazer que implicam exposição mais frequente às coleções hídricas com presença dos planorbídeos.

O tratamento quimioterápico com Praziquantel, em dose única, vem sendo utilizado como forma de combate à esquistossomose, com resultados instantâneos otimistas, reduzindo significativamente as formas graves e letais e a prevalência em determinadas áreas 21,22; apesar disto, observou-se que 33,2\% (334/1.006) dos pacientes positivos diagnosticados na área de estu-
Tabela 2

Contribuição do qui-quadrado na avaliação da importância de cada variável na dimensão 1 da análise de correspondência múltipla no grupo cluster primário.

\begin{tabular}{|c|c|c|}
\hline Variáveis & \multicolumn{2}{|c|}{ Cluster primário } \\
\hline \multicolumn{3}{|l|}{ Idade (anos) } \\
\hline $0-14$ & 31,3 & $(+)$ \\
\hline $15-29$ & 2,1 & $(+)$ \\
\hline$>29$ & 17,4 & \\
\hline \multicolumn{3}{|l|}{ Sexo } \\
\hline Masculino & 0,81 & $(+)$ \\
\hline Feminino & 1,5 & \\
\hline \multicolumn{3}{|l|}{ Ano } \\
\hline 2006 & 259,9 & $(+)$ \\
\hline 2007 & 62,9 & \\
\hline 2008 & 108,7 & \\
\hline \multicolumn{3}{|l|}{ Chuva (mm) } \\
\hline 0-100 & 0,0 & \\
\hline $101-200$ & 15,1 & \\
\hline$>200$ & 29,6 & $(+)$ \\
\hline \multicolumn{3}{|c|}{ Distância da água (m) } \\
\hline $0-50$ & 1,4 & $(+)$ \\
\hline $51-100$ & 3,8 & $(+)$ \\
\hline$>100$ & 9,3 & \\
\hline
\end{tabular}

do não compareceram às unidades de saúde para ser medicados, o que acaba contribuindo para a persistência da doença no município. Dessa forma, é premente a necessidade da realização de programas educativos nas escolas e nas comunidades reforçando não só a profilaxia, mas também a importância do tratamento para a redução da prevalência da doença no município.

Por se tratar de dados secundários, um dos principais problemas metodológicos deste estudo foi o georreferenciamento dos dados. Houve uma perda de $20,8 \%$ do georreferenciamento das residências dos casos positivos, em função do preenchimento incompleto do campo referente ao endereço nas fichas cadastrais e pelo fato dos moradores mudarem de residência com frequência.

A vasta extensão territorial dos municípios, de um modo geral, tem aparecido como um grande fator limitante no cenário do controle das doenças endêmicas, em virtude da demanda de um grande número de recursos humanos e custos operacionais envolvidos no processo. Assim, a utilização das análises espaciais vem se firmando no campo da saúde pública por se distinguirem das demais técnicas empregadas em análise estatística, possibilitando a identificação 
de áreas de risco e de grupos prioritários para a intervenção.

Os resultados das análises espaciais sugerem que a distribuição de casos de esquistossomose mansônica no Município de Lauro de Freitas segue um padrão tendencial para a formação de aglomerados. Ao comparar os resultados dos dois métodos de análises espaciais utilizados neste artigo, verificamos que o de Kulldorff \& Nagarwalla 14 mostrou-se extremamente útil ao confirmar que os achados de Kernel podem ser apropriados para os serviços de saúde do município, apesar de caracterizar-se como um método subjetivo e exploratório que depende da percepção do pesquisador na definição de parâmetros.

O método de varredura espaço-temporal proposto por Kulldorff \& Nagarwalla 14 identificou quatro grupos de aglomerados com forte significância estatística. Outros aglomerados foram encontrados, mas não entraram na faixa de significância maior que 95\%, estipulada pelo método de replicação de Monte Carlo, tendo, portanto, a probabilidade de ocorrência atribuída ao acaso.

O esperado seria que a análise de varredura espaço-temporal fosse capaz de localizar aglomerados em todos os anos de estudo. No entanto, somente foram detectados aglomerados nos anos de 2006 e 2008, provavelmente em função do maior número de casos registrados nesses anos. Essa mesma tendência foi observada em outros estudos, como o de Carneiro et al. ${ }^{23}$ que detectou aglomerados de riscos espaço-temporais para a leishmaniose visceral em 26 municípios da região centro-leste do Estado da Bahia apenas nos anos de 1996 e 2001, apesar de o período de estudo ter sido entre os anos de 1994 a 2004.

Apesar de os indicadores socioeconômicos serem positivos para todos os setores censitários do município no que concerne à proporção de domicílios com abastecimento de água via rede geral, à proporção de domicílios com sanitário, à proporção de domicílios com destino do lixo coletado e à proporção de pessoas responsáveis pelos domicílios alfabetizadas, ultrapassando a casa dos $80 \%$ das moradias, observou-se in loco que a maioria dos endereços georreferenciados apresentava fatores de susceptibilidade para a ocorrência da doença, como presença de habitações em áreas próximas às aguadas com a presença dos caramujos do gênero Biompha- laria, precariedade da qualidade de vida, baixa escolaridade e escasso lazer. Tais evidências são concordantes com outras pesquisas pertinentes ao tema $24,25,26$.

À semelhança do que observamos no Município de Lauro de Freitas, a grande maioria dos trabalhos que abordam os fatores socioeconômicos tem mostrado que o avanço e a disseminação da esquistossomose nas cidades brasileiras estão diretamente relacionados com a pobreza e com a forma de ocupação e organização do espaço $27,28,29$. No nosso estudo observou-se que os estratos sociais com maior risco de contrair a doença possuem 9,3\% dos domicílios com abastecimento de água via poço ou nascente; $8,3 \%$ dos domicílios sem sanitário; $12,4 \%$ do lixo jogado em terreno baldio; $18 \%$ das pessoas responsáveis pelos domicílios não alfabetizadas; $42,2 \%$ com o Ensino Fundamental representando o curso mais elevado; e $60,1 \%$ com rendimento mensal de até três salários mínimos.

A associação observada entre a doença, no grupo pertencente ao aglomerado primário, e escolares com faixa etária entre 0 e 14 anos, reforça o postulado da OMS em que os programas de controle de parasitoses devem dar prioridade às crianças em idade escolar, em função da alta vulnerabilidade dos jovens no risco de infecção 30 .

Apesar da análise de correlação ter mostrado, no período de estudo, que a ocorrência da doença independe da precipitação pluviométrica, observou-se que precipitações acima de 200mm apareceram como fator de importância para o aglomerado primário. Pode-se inferir que nos meses chuvosos os criadouros de moluscos transbordam, possibilitando a invasão de ruas e quintais pelos planorbídeos do gênero Biomphalaria com consequente promoção da infecção em pessoas sadias.

A abordagem metodológica utilizada neste estudo mostrou-se eficiente para o Programa de Controle da Esquistossomose desenvolvido no Município de Lauro de Freitas por identificar e delimitar as áreas de risco e as populações prioritárias para as ações de intervenção, possibilitando um processo de remodelação e adequação das estratégias do programa de controle da endemia, otimizando suas atividades e recursos, e minimizando os danos às populações expostas aos riscos. 


\section{Resumo}

A disseminação da esquistossomose mansônica vem desafiando o sistema de saúde brasileiro, deixando clara a necessidade da reavaliação das estratégias do programa de controle da endemia. O objetivo deste trabalho foi delimitar as áreas geográficas de risco para a esquistossomose em Lauro de Freitas, Bahia, Brasil, e estabelecer o perfil epidemiológico e socioeconômico da doença no município. Utilizou-se o estimador de densidade de Kernel para a identificação visual de aglomerados de casos e a análise de varredura espaçotemporal de Kulldorff \& Nagarwalla para a obtenção de aglomerados com significância estatística e mensuração do risco. As duas técnicas identificaram quatro áreas de risco para a doença no município, com indicadores socioeconômicos mais baixos que as áreas fora dos aglomerados. A análise de correspondência múltipla mostrou um perfil diferenciado nos pacientes positivos para a esquistossomose pertencentes ao aglomerado primário. As técnicas empregadas se configuram em uma importante aquisição metodológica para a vigilância e controle da doença no município.

Esquistossomose Mansoni; Análise Espacial; Zona de Risco

\section{Colaboradores}

L. L. Cardim participou na concepção, projeto, análise e interpretação de dados; redação do artigo; aprovação final da versão a ser publicada. A. S. Ferraudo e R. B. Reis colaboraram na análise e interpretação dos dados; revisão crítica relevante do conteúdo intelectual; aprovação final da versão a ser publicada. S. T. A. Pacheco participou da concepção e projeto; revisão crítica relevante do conteúdo intelectual; aprovação final da versão a ser publicada. M. M. N. Silva e D. D. M. T. Carneiro contribuíram na análise e interpretação dos dados; redação do artigo; aprovação final da versão a ser publicada. M. E. Bavia participou da concepção, projeto e análise e interpretação de dados; revisão crítica relevante do conteúdo intelectual; aprovação final da versão a ser publicada.

\section{Agradecimentos}

À Coordenação de Aperfeiçoamento de Pessoal de Nível Superior (CAPES) pelo auxílio financeiro; ao Departamento de Vigilância à Saúde da Secretaria Municipal de Saúde de Lauro de Freitas pela viabilidade de desenvolvimento do projeto e pelo apoio logístico; aos agentes de endemias e funcionários da Fundação Nacional de Saúde (FUNASA) do município pelo trabalho desenvolvido.

\section{Referências}

1. Neves DP. Parasitologia humana. 11a Ed. Rio de Janeiro: Editora Atheneu; 1999.

2. Bina JC, Prata A. Esquistossomose na área hiperendêmica de Taquarendi. I - Infecção pelo Schistosoma mansoni e formas graves. Rev Soc Bras Med Trop 2003; 36:211-6.

3. Fonseca FR, Saraiva TS, Freitas CC, Dutra LV, Monteiro AMV, Rennó CD, et al. Desenvolvimento de um índice hidrológico para aplicação em estudos de distribuição da prevalência de esquistossomose em Minas Gerais. In: Anais do Simpósio Brasileiro de Sensoriamento Remoto. Florianópolis: Instituto Nacional de Pesquisas Espaciais; 2007. p. 2589-95.

4. Departamento de Vigilância Epidemiológica, Secretaria de Vigilância em Saúde, Ministério da Saúde. Guia de vigilância epidemiológica. 7ạ Ed. Brasília: Ministério da Saúde; 2009.
5. Sistema Nacional de Vigilância em Saúde, Secretaria de Vigilância em Saúde, Ministério da Saúde. Relatório de situação, Bahia. Brasília: Ministério da Saúde; 2006.

6. Farias LMM, Resendes APC, Sabroza PC, SouzaSantos R. Análise preliminar do sistema de informação do Programa de Controle da Esquistossomose no período de 1999 a 2003. Cad Saúde Pública $2007 ; 23: 235-39$

7. Departamento de Vigilância Epidemiológica, Secretaria de Vigilância em Saúde, Ministério da Saúde. Guia de vigilância epidemiológica 2005. 6ạ Ed. Brasília: Ministério da Saúde; 2005.

8. Pellegini DCP. Análise espaço-temporal da leptospirose no Município do Rio de Janeiro (1995-1999) [Dissertação de Mestrado]. Rio de Janeiro: Escola Nacional de Saúde Pública, Fundação Oswaldo Cruz; 2002. 
9. Bavia ME, Carneiro DDMT, Gurgel HC, Madureira Filho C, Barbosa MGR. Remote sensing and geographic information system and risk of American Visceral Leishmaiasis in Bahia, Brazil. Parassitologia $2005 ;$ 47:165-9.

10. Clements ACA, Lwambo NJS, Blair L, Nyandindi U, Kaatano G, Kinung'hi S, et al. Bayesian spatial analysis and disease mapping: tools to enhance planning and implementation of a schistosomiasis control programme in Tanzania. Trop Med Int Health 2006; 11:490-503.

11. Instituto Brasileiro de Geografia e Estatística. Estimativa da população; 2009. http://ibge.gov.br/ home/estatistica/populacao/estimativa2009/ POP2009_DOU.pdf (acessado em 15/Ago/2009).

12. Flauzino RF, Souza-Santos R, Barcellos C, Gracie R, Magalhães MAFM, Oliveira RM. Heterogeneidade espacial da dengue em estudos locais, Niterói, RJ. Rev Saúde Pública 2009; 43:1035-43.

13. Silverman BW. Density estimation for statistics and data analysis. London: Chapman and Hall; 1986. (Monographs on Statistics and Applied Probability, 26).

14. Kulldorff M, Nagarwalla N. Spatial disease clusters: detection and inference. Stat Med 1995; 14:799810.

15. Hair JF, Anderson RE, Tatham RL, Black W. Análise multivariada de dados. 5a Ed. Porto Alegre: Bookman: 2005.

16. World Health Organization. The control of schistosomiasis. Geneva: World Health Organization; 1985.

17. Doenhoff MJ, Chiodini PL, Hamilton JV. Specific and sensitive diagnosis of schistosome infection: can it be done with antibodies? Trends Parasitol 2004; 20:35-9.

18. Enk MJ, Lima ACL, Massara CL, Coelho PMZ, Schall VT. A combined strategy to improve the control of Schistosoma mansoni in areas of low prevalence in Brazil. Am J Trop Med 2008; 78:140-6.

19. Nomura YM, Camargos MO, Bichara CNC, Rodrigues IRC. Esquistossomose mansônica em Carajás, Pará, Brasil: estudo retrospectivo realizado no hospital Yutaka Takeda. Cad Saúde Colet (Rio J.) 2007; 15:531-42.

20. Vasconcelos CH, Cardoso PCM, Quirino WC, Massara CL, Amaral GL, Cordeiro R, et al. Avaliação das medidas de controle da esquistossomose mansoni no Município de Sabará, Minas Gerais, Brasil, 1980-2007. Cad Saúde Pública 2009; 25:997-1006.
21. Santana VS, Teixeira MG, Santos CP, Andrade CAR. Efetividade do programa de comunicação em saúde no controle da infecção por Schistosoma mansoni em algumas áreas do Estado da Bahia. Rev Soc Bras Med Trop 1997; 30:447-56.

22. Carvalho EMF, Acioli MD, Branco MA, Costa AMF, Cesse EAP, Andrade AG, et al. Evolução da esquistossomose na Zona da Mata Sul de Pernambuco. Epidemiologia e situação atual: controle ou descontrole. Cad Saúde Pública 1998; 14:787-95.

23. Carneiro DD, Bavia ME, Rocha WJ, Tavares AC, Cardim LL, Alemayehu B. Application of spatiotemporal scan statistics for the detection of areas with increased risk for American visceral leishmaniasis in the state of Bahia, Brazil. Geospat Health 2007; 2:113-26.

24. Oliveira AS, Santos JF. Aspectos epidemiológicos da esquistossomose mansônica nos bairros Novo Horizonte e Campo Limpo, Feira de Santana, Bahia. Sittentibus Ciências Biológicas 2002; 2:69-72.

25. Grazzinelli A, Melendez GV, Crawford SB, Loverde PT, Oliveira RC, Kloos H. Socioeconomic determinants of schistosomiasis in a poor rural area in Brazil. Acta Trop 2006; 99:260-71.

26. Cardim LL, Bavia ME, Ferraudo AS, Carneiro DDMT, Silva MMN, Brito VS, et al. Avaliação da esquistossomose mansônica mediante as geotecnologias e técnicas multivariadas no Município de Jacobina, Bahia. Rev Baiana Saúde Pública 2008; 32:29-42.

27. Ximenes R, Southgate B, Smith PG, Neto LG. Socioeconomic determinants in a urban area in the Northeast of Brazil. Rev Panam Salud Pública 2003; 14:409-21.

28. Peixoto LE, Machado MI. Vigilância ambiental em saúde: ocorrência de Biomphalaria peregrina e B. schrammi em áreas de influência da usina hidrelétrica de Miranda, Minas Gerais. In: Anais do 23o Congresso Brasileiro de Engenharia Sanitária e Ambiental. http://www.bvsde.paho.org/bvsacd/ abes23/VII-051.pdf (acessado em 19/Abr/2009).

29. Anaruma Filho F, Santos RF. Indicadores da relação entre estrutura da paisagem, degradação ambiental e esquistossomose mansoni. In: Anais do 8o Congresso de Ecologia do Brasil. http://www. seb-ecologia.org.br/viiiceb/pdf/1672.pdf (acessado em 15/Jul/2009).

30. World Health Organization. The control of schistosomiasis. Second report of the WHO Expert Committee. Geneva: World Health Organization; 1993.

Recebido em 23/Set/2010

Versão final reapresentada em 14/Fev/2011

Aprovado em 05/Abr/2011 